\title{
Genetic Counseling Referral Rates in Long-Term Survivors of Triple-Negative Breast Cancer
}

\author{
Carlos H. Barcenas, MD, MS a,"; Maryam N. Shafaee, MD ${ }^{b, *}$; Arup K. Sinha, PhDc; \\ Akshara Raghavendra, MDa; Babita Saigal, BSª Rashmi K. Murthy, MDª; Ashley H. Woodson, MS; \\ and Banu Arun, MDa
}

\begin{abstract}
Background: Inherited $B R C A$ gene mutations (pathogenic variants) cause $10 \%$ of breast cancers. $B R C A$ pathogenic variants predispose carriers to triple-negative breast cancer (TNBC); around $30 \%$ of patients with TNBC carry BRCA pathogenic variants. The 2018 NCCN Guidelines for Genetic/Familial High-Risk Assessment: Breast and Ovarian recommend genetic counseling referrals for patients with TNBC diagnosed at age $\leq 60$ years. This study sought to describe genetic counseling referral patterns among long-term TNBC survivors at The University of Texas MD Anderson Cancer Center. Methods: This single-institution retrospective analysis of female long-term (disease-free for $\geq 5$ years) TNBC survivors sought to determine the rate of genetic counseling referral among patients diagnosed at age $\leq 60$ years between 1992 and 2008. Patients who underwent treatment and surveillance visits at our institution and were followed until 2017 were included. We collected $B R C A$ pathogenic variant status among tested patients. Descriptive statistical methods and a univariate analysis were used to identify patient characteristics associated with genetic counseling referral. Results: We identified 646 female long-term TNBC survivors with a median age at diagnosis of 47 years. Of these, $245(38 \%)$ received a recommendation for a genetic counseling referral. Among those referred, $156(64 \%)$ underwent genetic testing, and 35\% of those tested had BRCA pathogenic variants. Interestingly, among those referred, $20 \%$ declined genetic testing. The rate of genetic referrals improved over time, from $25 \%$ among TNBC survivors whose last surveillance visit was between 2011 and 2013 to $100 \%$ among those whose last surveillance visit was between 2014 or later. Younger age and premenopausal status at diagnosis and a family history of breast or ovarian cancer were associated with an increased rate of referral for genetic counseling. Conclusions: Among long-term TNBC survivors, the rate of referral to genetic counseling increased over time, and among those tested, $35 \%$ carried a $B R C A$ pathogenic variant. Survivorship care provides an excellent opportunity to refer eligible patients for genetic counseling.
\end{abstract}

J Natl Compr Canc Netw 2018;16(5):518-524 doi: 10.6004/jnccn.2018.7002

\section{Background}

Inherited germline mutations, currently identified in the genetics terminology as pathogenic variants, cause $5 \%$ to $10 \%$ of breast cancers. ${ }^{1}$ Triple-negative breast cancers (TNBC), which lack expression of estrogen and progesterone receptors and have normal expression of

aDepartment of Breast Medical Oncology, The University of Texas MD Anderson Cancer Center, Houston, Texas; 'bester \& Sue Smith Breast Center, Baylor College of Medicine, Houston, Texas; and 'Department of Biostatistics, Yale School of Public Health, New Haven, Connecticut. *These authors contributed equally.

Submitted October 6, 2017; accepted for publication January 2, 2018. The authors have disclosed that they have no financial interests, arrangements, affiliations, or commercial interests with the manufacturers of any products discussed in this article or their competitors.

This study was supported in part by the NIH through Cancer Center
HER2, account for $10 \%$ to $17 \%$ of all invasive breast cancers. ${ }^{2}$ Up to $30 \%$ of women who have a diagnosis of TNBC carry a BRCA gene pathogenic variant, ${ }^{3,4}$ and this phenomenon is particularly more predominant among those who harbor a BRCA1 pathogenic variant compared with BRCA2 carriers who do not have

Support Grant P30 CA016672 and by the Young Breast Cancer Survivors Program (104029) from MDACC. The content is solely the responsibility of the authors and does not necessarily represent the official views of the $\mathrm{NCl}$ or $\mathrm{NIH}$.

Author contributions: Study concept and design: Barcenas, Shafaee. Data acquisition: Barcenas, Shafaee, Raghavendra, Saigal, Murthy, Woodson. Data analysis and interpretation: Barcenas, Shafaee, Sinha, Raghavendra, Arun. Manuscript preparation and final approval: All authors.

Correspondence: Carlos H. Barcenas, MD, MS, Department of Breast Medical Oncology, The University of Texas MD Anderson Cancer Center, 1515 Holcombe Boulevard, Unit 1358, Houston, TX 77030.

Email: chbarcenas@mdanderson.org 
the same excess risk of developing TNBC. ${ }^{5}$ As many as $57 \%$ of breast cancers diagnosed in women who carry a deleterious BRCA1 pathogenic variant are of the TNBC subtype. ${ }^{6}$ Women who have a deleterious BRCA1 or BRCA2 germline pathogenic variant not only have a high risk of developing a second primary breast cancer ( $50 \%$ at 25 years) but also have a $40 \%$ lifetime risk of developing ovarian cancer. ${ }^{7}$ Additionally, prostate and pancreatic cancers have been reported at higher rates in BRCA pathogenic variant carriers. ${ }^{8}$ Hence, the importance of a timely diagnosis of these germline pathogenic variants cannot be overemphasized because preventive measures for patients or their families can be applied.

National guidelines regarding referral to genetic counseling and testing for patients with cancer have evolved over time. The NCCN Clinical Practice Guidelines in Oncology (NCCN Guidelines) for Genetic/Familial High-Risk Assessment: Breast and Ovarian, among other established criteria, recommend that women with a history of TNBC at age $\leq 60$ years receive a referral for genetic counseling with possible genetic testing. ${ }^{9}$ These referral criteria were initially published by NCCN in May 2010.1 Therefore, TNBC survivors diagnosed at age $\leq 60$ years would be eligible for a genetic counseling referral under current criteria. However, many long-term TNBC survivors were diagnosed before the NCCN Guidelines were initially published and widely disseminated, and therefore may not have been offered a referral for genetic counseling at initial diagnosis or at subsequent surveillance visits. ${ }^{11}$

In our practice within a breast cancer survivorship clinic at The University of Texas MD Anderson Cancer Center (MDACC), we anecdotally observed that some TNBC survivors who had been diagnosed at a young age and were being followed with routine surveillance visits had not undergone genetic counseling or testing. Hence, in this retrospective study, we sought to understand referral patterns for genetic counseling and testing among long-term TNBC survivors at MDACC. We aimed to determine factors associated with such referrals and to seek opportunities for quality improvement and increasing the rate of referral to genetic counseling.

\section{Methods}

We conducted a single-institution retrospective analysis of female long-term TNBC survivors, defined as being disease-free for a minimum of 5 years after diagnosis. The primary outcome of interest was documentation of a formal recommendation from the medical provider for a genetic counseling session. Using the prospectively maintained Breast Cancer Database Management System housed in the Department of Breast Medical Oncology at MDACC, we identified women diagnosed with stage I-III TNBC between 1992 and 2008, were aged $\leq 60$ years at diagnosis, were alive and cancer-free for at least 5 years from the date of diagnosis, and had undergone surveillance visits up to 2017. To avoid selection bias, we included those who had received their initial treatment and subsequent surveillance visits at MDACC and excluded those who had presented only for an initial consultation or a second opinion. ${ }^{12}$

We reviewed the electronic medical records of these women and extracted data on demographic characteristics, including ethnicity/race, menopausal status, family history of breast and ovarian cancer in first- and second-degree relatives, and tumor characteristics, including tumor stage and grade. At MDACC, a genetic counseling session includes a comprehensive assessment of a personal and family history to ensure patients receive genetic testing recommendations as established by the most up-to-date clinical care for any hereditary cancer syndrome. For those who underwent genetic testing, BRCA test results were noted if available. We compared rates of genetic referrals among those who had undergone their last surveillance visit up to 2010, which is when NCCN initially published these specific criteria, ${ }^{10}$ and used this rate as a baseline relative control, versus those who had undergone such a surveillance visit during 2011 and later, to determine the influence of the initial NCCN Guidelines in the rates of referrals. We also compared rates of referrals by the year of diagnosis.

We used descriptive statistics to analyze the characteristics of these patients and a univariate analysis to identify variables associated with an increased rate of genetic counseling referral. Statistical tests were performed using a 2-sided significance level of 0.05 , by using SAS 9.4 (SAS Institute Inc.) and STATA version 12.0 (StataCorp LP). This retrospective study was conducted under Institutional 
Barcenas et al

Review Board-approved protocol PA13-0424, and a waiver of informed consent was obtained.

\section{Results}

We identified 646 female long-term TNBC survivors, who had a median age at diagnosis of 47 years. Among them, 64\% were white, 18\% were African American, and $13 \%$ were of Hispanic ethnicity (Table 1). Documentation of a formal recommendation from the medical provider for a referral to a genetic counseling session with potential for genetic testing was found in the medical records of 245 TNBC survivors (38\%), among whom 59\% had no family history of breast or ovarian cancer, whereas $23 \%$ had a positive family history in a first-degree relative and $18 \%$ in a second-degree relative. Among those referred for genetic counseling, 156 (64\%) underwent genetic testing and had results available in the medical record. Among these, 55 (35\%) were found to have a deleterious BRCA germline pathogenic variant ( 38 with a $B R C A 1$ pathogenic variant, 15 with a BRCA2 pathogenic variant, and 2 with pathogenic variants in both BRCA1 and BRCA2).
Among the 55 long-term TNBC survivors who carried a BRCA pathogenic variant, $78 \%$ were diagnosed at age $<50$ years. In regard to timing to genetic testing among these 55 women, $2(4 \%)$ had been tested before their diagnosis of TNBC, 17 (31\%) were tested at time of diagnosis, 18 (33\%) had been tested between years 1 to 4 after their diagnosis, and $14(25 \%)$ had been tested after 5 years of their diagnosis of TNBC; of note, 4 TNBC survivors (7\%) did not have available documentation on the exact date of the genetic testing.

In reference to the clinical impact of finding a BRCA pathogenic variant in these 55 female TNBC patients, 21 (38\%) underwent a prophylactic contralateral mastectomy and a prophylactic bilateral salpingo-oophorectomy (BSO) after testing positive; $14(26 \%)$ underwent a prophylactic BSO after genetic testing, wherein some of these patients had a diagnosis of synchronous or metachronous bilateral breast cancer; 6 (11\%) underwent a contralateral prophylactic mastectomy followed by a high-risk ovarian cancer screening program including serial measurements of the CA-125 tumor marker and a vaginal ultrasound; and 11 (20\%) did not have any

\begin{tabular}{|c|c|c|c|c|}
\hline Characteristics & $\begin{array}{c}\text { All } \\
(\mathrm{N}=646)\end{array}$ & $\begin{array}{l}\text { Referred for Genetic } \\
\text { Counseling } \\
(\mathrm{N}=245)\end{array}$ & $\begin{array}{l}\text { Not Referred } \\
\quad(\mathrm{N}=401)\end{array}$ & $P$ Value \\
\hline Age at diagnosis, $y$ & & & & $<.001$ \\
\hline Mean (SD) & $46(8.0)$ & $44(8.2)$ & $48(7.5)$ & \\
\hline Median (1st-3rd quartile) & $47(41-53)$ & $45(39-50)$ & $49(42-54)$ & \\
\hline Race/Ethnicity, n (\%) & & & & .427 \\
\hline White & $414(64.1)$ & $162(66.1)$ & $252(62.8)$ & \\
\hline African American & $115(17.8)$ & 39 (15.9) & $76(19.0)$ & \\
\hline Hispanic & $82(12.7)$ & 34 (13.9) & $48(12.0)$ & \\
\hline Other & $35(5.4)$ & $10(4.1)$ & $25(6.2)$ & \\
\hline Menopausal status, n (\%) & & & & .014 \\
\hline Postmenopausal & $322(49.8)$ & $107(43.7)$ & $215(53.6)$ & \\
\hline Premenopausal & $296(45.8)$ & $130(53.1)$ & $166(41.4)$ & \\
\hline Unknown & $28(4.3)$ & $8(3.3)$ & $20(5.0)$ & \\
\hline Disease stage, ${ }^{a} \mathrm{n}(\%)$ & & & & .779 \\
\hline 1 & $205(31.7)$ & $74(30.2)$ & $131(32.7)$ & \\
\hline II & $340(52.6)$ & $133(54.3)$ & $207(51.6)$ & \\
\hline III & $101(15.6)$ & $38(15.5)$ & $63(15.7)$ & \\
\hline Tumor grade, n (\%) & & & & .424 \\
\hline $1-I I$ & $56(8.7)$ & $17(6.9)$ & $39(9.7)$ & \\
\hline III & $572(88.5)$ & $222(90.6)$ & $350(87.3)$ & \\
\hline Unknown & $18(2.8)$ & $6(2.4)$ & $12(3.0)$ & \\
\hline Family history of breast and/or ovarian cancer, $\mathrm{n}(\%)$ & & & & $<.001$ \\
\hline None & $545(84.4)$ & $144(58.8)$ & $401(100)$ & \\
\hline In a first-degree relative & $56(8.7)$ & $56(22.9)$ & $0(0)$ & \\
\hline In a second-degree relative & $45(7.0)$ & 45 (18.4) & $0(0)$ & \\
\hline
\end{tabular}

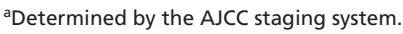


prophylactic surgery due to either a diagnosis of bilateral breast cancer and ovarian cancer $(n=9)$ or because they were morbidly obese and thus not surgical candidates $(n=2)$. Of these 55 patients, $3(5 \%)$ had undergone $\mathrm{BSO}$ prior to genetic testing for a nonmalignant reason. The BRCA pathogenic variant carriers who did not undergo a bilateral mastectomy were recommended to follow a high-risk breast cancer program with serial mammograms and breast MRI.

Interestingly, the medical records indicated that $20 \%$ of the long-term TNBC survivors who had received a recommendation for a referral for genetic counseling declined genetic counseling or testing (Figure 1). In a small proportion (3\%) of cases, the genetic counselor did not recommend testing, whereas another $2 \%$ of survivors did not pursue testing because of lack of medical insurance coverage. There were 5 TNBC survivors (2\%) who, according to documentation, underwent genetic counseling and testing, but the results were unavailable in the medical records. Another 9\% of those who were recommended to undergo genetic counseling did not, for reasons that could not be determined from the available documentation.

The rate of referral for genetic counseling markedly improved over time. We established a baseline relative control referral rate of 15\% (23/150) among long-term TNBC survivors whose most recent docu- mented surveillance clinic visit had occurred up until 2010, whereas this rate was 25\% (90/364) among those whose most recent surveillance clinic visit occurred between 2011 and 2013, and 100\% ( $n=132)$ among those who had such surveillance visits in 2014 and later. Conversely, the year of diagnosis did not appear to have much influence on the rates of genetic referrals: 33\% (48/146) among long-term TNBC survivors diagnosed from 1992-2000 compared with 39\% (197/500) among those diagnosed from 2001-2008.

Patient characteristics associated with receiving a genetic counseling referral were younger age at diagnosis, premenopausal status at diagnosis, and positive family history of breast and/or ovarian cancer (Table 2). No associations were found between referral for genetic counseling and race/ethnicity, disease stage, or tumor grade.

\section{Discussion}

We found that $38 \%$ of long-term TNBC survivors who were diagnosed at age $\leq 60$ years and who received their initial treatment and continued surveillance care at MDACC received a recommendation for referral to genetic counseling. Among these survivors, 64\% underwent genetic testing. Among those tested, $35 \%$ were found to have a BRCA germline pathogenic variant, which is in line with find-

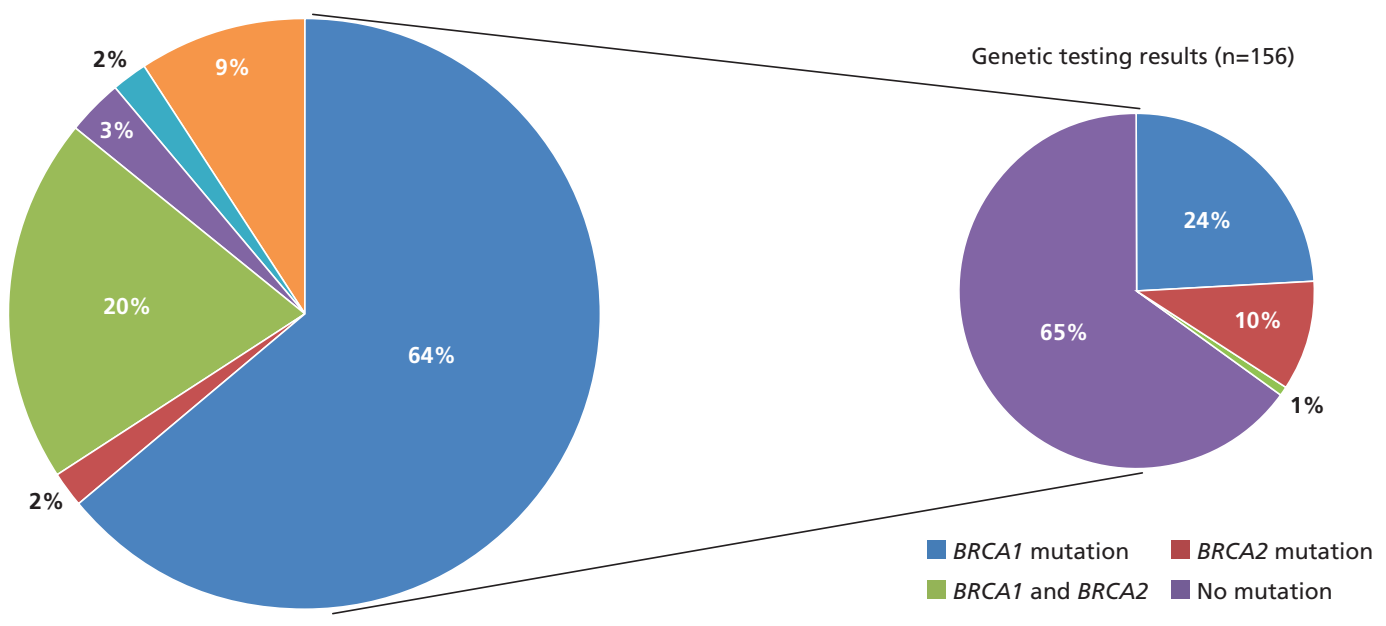

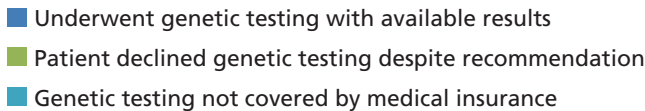

Underwent genetic testing with available results

Genetic testing not covered by medical insurance
Underwent genetic testing but results are unavailable

Genetic testing not recommended during counseling

Reasons for not undergoing genetic testing not documented

Figure 1. Outcome of referral to genetic counseling ( $N=245)$. 
Barcenas et al

\begin{tabular}{|c|c|c|c|}
\hline Characteristics $^{\mathrm{a}}$ & Odds Ratio & $95 \% \mathrm{Cl}$ & $P$ Value \\
\hline Age at diagnosis & 0.94 & $0.92-0.96$ & $<.001$ \\
\hline \multicolumn{4}{|l|}{ Race/Ethnicity } \\
\hline White & Ref & & \\
\hline African American & 0.80 & $0.52-1.23$ & .308 \\
\hline Hispanic & 1.10 & $0.68-1.78$ & .693 \\
\hline Other & 0.62 & $0.29-1.33$ & .221 \\
\hline \multicolumn{4}{|l|}{ Menopausal status } \\
\hline Postmenopausal & Ref & & \\
\hline Premenopausal & 1.57 & $1.14-2.18$ & .006 \\
\hline \multicolumn{4}{|l|}{ Disease stage } \\
\hline 1 & Ref & & \\
\hline II & 1.14 & $0.79-1.63$ & .482 \\
\hline III & 1.07 & $0.65-1.75$ & .794 \\
\hline \multicolumn{4}{|l|}{ Tumor grade } \\
\hline I-II & Ref & & \\
\hline III & 1.46 & $0.80-2.64$ & .216 \\
\hline
\end{tabular}

a Family history of breast and/or ovarian cancer was not included in this analysis because an odds ratio was not calculable.

ings from previous studies of patients with TNBC. ${ }^{3}$ Univariate analysis showed that younger age, premenopausal state at diagnosis, and family history of breast and/or ovarian cancer were associated with an increased rate of receiving a referral. A multivariable analysis was not possible because a premenopausal state was considered to be correlated with a younger age at diagnosis, and because among the 401 longterm TNBC survivors who did not receive a formal recommendation for a referral to genetic counseling, none had a family history of breast and/or ovarian cancer. Therefore, an odds ratio could not be calculated for the family history variable.

Other studies have shown that the rates of genetic testing among patients with newly diagnosed breast cancer who were eligible for referral based on NCCN criteria were as high as $53 \%$ among those diagnosed in 2013 and 2014. ${ }^{13}$ However, in a recent population-based study, $<20 \%$ of women with a history of breast and/or ovarian cancer who met NCCN eligibility criteria had actually undergone genetic testing; the authors estimated that $>1$ million eligible women had not undergone genetic testing. ${ }^{14}$ The care of long-term TNBC survivors could be substantially affected by genetic test results, even long after the course of their initial oncologic treatments. Many patients may have undergone unilateral or partial mastectomies, and many may still have their ovaries, increasing the risk of recurrence or secondary cancers. Additionally, results of a positive BRCA test may prompt patients' family members to seek genetic testing to determine their pathogenic variant status and, if positive, to seek preventive care. ${ }^{11}$

We found that the rate of referral for genetic counseling markedly increased over time, from a baseline control of $15 \%$ until 2010 , to $25 \%$ from 2011-2013, to 100\% from 2014 and onward; in May 2010, the first NCCN Guidelines that established clear criteria for genetic counseling referral in patients with breast cancer were published. ${ }^{10}$ Improvement in the rate of genetic counseling referral over time speaks to the likely influence of the NCCN Guidelines in changing clinical practice. This outcome is consistent with other reports in the literature. Using an insurance claims-based approach, one study showed that among almost 27,000 young breast cancer survivors who had been diagnosed at age $\leq 45$ years, BRCA testing rates increased each year from 2005 to 2012, increasing substantially in the past decade. ${ }^{15}$ Another study, a cross-sectional analysis of a prospective cohort, showed that the rates of BRCA pathogenic variant testing in almost 900 women aged $\leq 40$ years at breast cancer diagnosis increased between 2006 and 2013. ${ }^{16}$ A recent study from a university-based hospital reported a significant increase in their genetic referral rates from $26 \%$ to $52 \%$ after the implementation of a multidisciplinary Heredofamilial Cancer Unit, which offers cancer risk assessment services provided by a medical oncologist trained in human genetics and genetic counseling, and access to a multidisciplinary team of breast cancer providers including a gynecologist, a radiologist, an oncology nurse, and a psychologist. ${ }^{17}$

Although our study focused on women with TNBC who were diagnosed at age $\leq 60$ years, the NCCN Guidelines have established additional criteria for recommending a referral for genetic risk evaluation. These criteria include patients with breast cancer with a known cancer susceptibility gene pathogenic variant within their family, those diagnosed at $\leq 50$ years of age, individuals who have 2 breast cancer primaries, male patients with breast cancer, patients with a significant family history of breast or ovarian cancer, individuals of Ashkenazi Jewish descent, and individuals with a personal and/ or family history of cancer in other sites, such as pancreas and prostate, among other sites, following specific criteria outlined in the guideline. ${ }^{9}$ In addition, family history of cancer can change over time-on 
diagnosis, a newly diagnosed patient could be the index case in the family, but after a period of time, other family members may also develop breast or ovarian cancer, which could be indicative of a deleterious germline pathogenic variant in the family. Hence, it is important for medical providers who care for breast cancer survivors to review and update the family history of cancer during each clinical encounter. ${ }^{18}$ A significant number of long-term breast cancer survivors could meet the eligibility criteria for genetic counseling only many years after diagnosis. In our analysis, $59 \%$ of patients referred for genetic counseling did not have a positive family history of breast cancer. However, as expected, the BRCA pathogenic variant rate was significantly higher in those with a first- or second-degree family history of breast and/or ovarian cancer than in those without such a family history.

Interestingly, $20 \%$ of the referred breast cancer survivors in our cohort declined genetic testing. Reasons why patients decline genetic counseling and testing have been documented previously and include a lack of knowledge about breast cancer genetics, fear of stigmatization, anticipation of negative emotions as a result of undergoing testing, and high levels of family-related guilt. ${ }^{19,20}$ Additionally, some patients who refuse genetic testing may feel that results of the testing are no longer relevant to them or may be afraid of the results after a relative's objection. ${ }^{21}$ Some barriers to genetic counseling and testing for patients who meet the NCCN criteria for genetic testing have been addressed by the Genetic Information Nondiscrimination Act (GINA) of 2008, a federal law that protects consumers from discrimination by health insurers and employers based on genetic information. ${ }^{22,23}$ Our study found that among the 401 long-term TNBC survivors who did not receive a formal recommendation for genetic counseling, none had a family history of breast and/ or ovarian cancer. Hence, an alternative explanation for the increase in genetic referrals over time was likely the improved insurance coverage after publication of the GINA. Nonetheless, the screening interventions that may be required for patients with positive genetic test results can be cost-prohibitive; insurance coverage of preventive measures for carriers of deleterious germline pathogenic variants is not mandated by the Affordable Care Act, and these costs can disproportionately affect low-income minority populations. ${ }^{24}$ Furthermore, many patients with breast cancer undergo genetic testing without ever seeing a genetic counselor, and the growing use of multigene panels adds more challenges and complexity for both medical providers and patients. ${ }^{25}$ Knowledge of other inherited breast cancer related risks, such as pathogenic variants in PALB2, ATM, and CHEK2, could warrant rereferral for genetic counseling and potential expanded multigene panel testing among patients with TNBC who had previously tested negative for a BRCA pathogenic variant. ${ }^{26}$ However, limited data exist on the prevalence of TNBC among carriers of pathogenic variants in genes beyond BRCA1 and BRCA2.

A survey of $>1,500$ women with nonmetastatic breast cancer diagnosed from 2005 to 2007 found that $35 \%$ had a strong desire for genetic testing. ${ }^{27}$ This desire was more common among younger women, and minority patients' need for discussing genetic testing with their medical providers was more likely to go unmet. ${ }^{27}$ Racial disparities in BRCA testing have been documented; rates of BRCA testing are substantially lower among African American women with breast cancer versus white women. ${ }^{28}$ Our study showed that survivors who were younger or premenopausal at diagnosis or had a significant family history of breast and/or ovarian cancer more often received a recommendation for genetic counseling. We did not observe any differences in recommendation rates based on race or ethnicity. Differences in the source of the study populations and methodology could explain these differences among studies.

This study has the inherent limitations of a retrospective review of medical records. Some information was not available for all individuals, and the reasons for a medical provider not recommending a genetic referral, despite managing an eligible patient, are not always well-documented in the medical record. It is also possible that family history is underreported and not probed in a consistent manner across providers. We believe that these limitations do not greatly affect the results and conclusions of this study.

\section{Conclusions}

Among long-term TNBC survivors, the rate of genetic counseling referral has increased over time from a baseline of $15 \%$ among those whose last sur- 
veillance visit occurred in 2010 or earlier, to $25 \%$ among those followed between 2011-2013, to 100\% among those followed in 2014 or later. The marked increase in referrals was likely influenced by publication of the 2010 NCCN Guidelines, ${ }^{10}$ which was the first version that established clear criteria for genetic counseling referral in patients with breast cancer, and suggests the substantial influence of these guidelines in changing clinical practice. An alternative explanation for this increase in genetic referrals over time may have been the improved insurance coverage after publication of the GINA in 2008.22 In addition, a considerable number of long-term TNBC survivors are carriers of a deleterious BRCA germline pathogenic variant. With evolving national guidelines that recommend genetic counseling and risk assessment in women with a history of breast or ovarian cancer, the role of survivorship care in addressing this need is highlighted. Designing interventions to standardize genetic testing, increase referrals to genetic counseling, and improve education for healthcare providers and patients regarding the clinical utility of genetic counseling testing as recommended by the current NCCN Guidelines ${ }^{9}$ will likely improve the rate of genetic counseling referral among eligible breast cancer survivors.

\section{Acknowledgments}

The authors are very grateful to Amy Ninetto, $\mathrm{PhD}$, from the Department of Scientific Publications of MD Anderson Cancer Center who edited the manuscript.

\section{References}

1. Couch FJ, Nathanson KL, Offit K. Two decades after BRCA: setting paradigms in personalized cancer care and prevention. Science 2014;343:1466-1470.

2. Bayraktar S, Gutierrez-Barrera AM, Liu D, et al. Outcome of triplenegative breast cancer in patients with or without deleterious BRCA mutations. Breast Cancer Res Treat 2011;130:145-153.

3. Wong-Brown MW, Meldrum CJ, Carpenter JE, et al. Prevalence of BRCA1 and BRCA2 germline mutations in patients with triple-negative breast cancer. Breast Cancer Res Treat 2015;150:71-80.

4. Kwon JS, Gutierrez-Barrera AM, Young D, et al. Expanding the criteria for BRCA mutation testing in breast cancer survivors. J Clin Oncol 2010;28:4214-4220.

5. Gonzalez-Angulo AM, Timms KM, Liu S, et al. Incidence and outcome of BRCA mutations in unselected patients with triple receptor-negative breast cancer. Clin Cancer Res 2011;17:1082-1089.

6. Atchley DP, Albarracin CT, Lopez A, et al. Clinical and pathologic characteristics of patients with BRCA-positive and BRCA-negative breast cancer. J Clin Oncol 2008;26:4282-4288.

7. Peshkin BN, Alabek ML, Isaacs C. BRCA1/2 mutations and triple negative breast cancers. Breast Dis 2010;32:25-33.

8. Petrucelli N, Daly MB, Feldman GL. Hereditary breast and ovarian cancer due to mutations in BRCA1 and BRCA2. Genet Med 2010;12:245-259.

9. Daly MB, Pilarski R, Berry M, et al. NCCN Guidelines: Genetic/Familial High-Risk Assessment: Breast and Ovarian. Version 1.2018. Accessed October 3, 2017. To view the most recent version of these guidelines, visit NCCN.org.

10. Daly MB, Axilbund JE, Buys S, et al. Genetic/familial high-risk assessment: breast and ovarian. J Natl Compr Canc Netw 2010;8:562-594.

11. Ruddy KJ, Risendal BC, Garber JE, Partridge AH. Cancer survivorship care: an opportunity to revisit cancer genetics. J Clin Oncol 2015;34:539541.

12. Sinha AK, Patel JR, Shen $Y$, et al. Location of receipt of initial treatment and outcomes in long-term breast cancer survivors. PLoS One 2017;12:e0170081.

13. Kurian AW, Griffith KA, Hamilton AS, et al. Genetic testing and counseling among patients with newly diagnosed breast cancer. JAMA 2017;317:531-534.

14. Childers CP, Childers KK, Maggard-Gibbons M, Macinko J. National estimates of genetic testing in women with a history of breast or ovarian cancer. J Clin Oncol 2017;35:3800-3806.

15. Kehl KL, Shen C, Litton JK, et al. Rates of BRCA1/2 mutation testing among young survivors of breast cancer. Breast Cancer Res Treat 2016;155:165-173.
16. Rosenberg SM, Ruddy KJ, Tamimi RM, et al. BRCA1 and BRCA2 mutation testing in young women with breast cancer. JAMA Oncol 2016;2:730-736.

17. Lobo M, Lopez-Tarruella S, Luque $S$, et al. Evaluation of breast cancer patients with genetic risk in a university hospital: before and after the implementation of a heredofamilial cancer unit [published online December 15, 2017]. J Genet Couns. doi: 10.1007/s10897-017-0187-3

18. Lu KH, Wood ME, Daniels M, et al. American Society of Clinical Oncology expert statement: collection and use of a cancer family history for oncology providers. J Clin Oncol 2014;32:833-840.

19. Thompson HS, Valdimarsdottir HB, Duteau-Buck C, et al. Psychosocial predictors of BRCA counseling and testing decisions among urban AfricanAmerican women. Cancer Epidemiol Biomarkers Prev 2002;11:15791585.

20. Sharma P, Klemp JR, Kimler BF, et al. Germline BRCA mutation evaluation in a prospective triple-negative breast cancer registry: implications for hereditary breast and/or ovarian cancer syndrome testing. Breast Cancer Res Treat 2014;145:707-714.

21. Schlich-Bakker KJ, ten Kroode HF, Warlam-Rodenhuis CC, et al. Barriers to participating in genetic counseling and BRCA testing during primary treatment for breast cancer. Genet Med 2007;9:766-777.

22. United States Congress House Committee on Energy and Commerce Subcommittee on Health. The Genetic Information Nondiscrimination Act: Hearing Before the Subcommittee on Health of the Committee on Energy and Commerce, House of Representatives, One Hundred Tenth Congress, first session, on H.R. 493. U.S.G.P.O.: for sale by the Supt. of Docs., U.S.G.P.O.; March 8, 2007. Washington, DC; 2008.

23. Hudson KL, Holohan MK, Collins FS. Keeping pace with the timesthe Genetic Information Nondiscrimination Act of 2008. N Engl J Med 2008;358:2661-2663.

24. Walcott FL, Dunn BK. Legislation in the genomic era: the Affordable Care Act and genetic testing for cancer risk assessment. Genet Med 2015;17:962-964.

25. Kurian AW, Li Y, Hamilton AS, et al. Gaps in incorporating germline genetic testing into treatment decision-making for early-stage breast cancer. J Clin Oncol 2017;35:2232-2239.

26. Offit K. Multigene testing for hereditary cancer: when, why, and how. J Natl Compr Canc Netw 2017;15:741-743.

27. Jagsi R, Griffith KA, Kurian AW, et al. Concerns about cancer risk and experiences with genetic testing in a diverse population of patients with breast cancer. J Clin Oncol 2015;33:1584-1591.

28. McCarthy AM, Bristol M, Domchek SM, et al. Health care segregation, physician recommendation, and racial disparities in BRCA1/2 testing among women with breast cancer. J Clin Oncol 2016;34:2610-2618. 